\title{
Simulating the hydraulic characteristics of the lower Yellow River by the finite-volume technique
}

\author{
Qing Wan, ${ }^{1}$ Hongtao Wan, ${ }^{1 *}$ Chenghu Zhou ${ }^{1}$ and Yingxiang $\mathrm{Wu}^{2}$ \\ ${ }^{1}$ The National Key Laboratory of Resource and Environment Information System (LREIS), Institute of Geography Science and Resource, \\ Chinese Academy of Sciences (CAS), Beijing 100101, People's Republic of China \\ ${ }^{2}$ Department of Applied Fluid Mechanics, Institute of Mechanics, CAS, Beijing 100080, People's Republic of China
}

\begin{abstract}
:
The finite-volume technique is used to solve the two-dimensional shallow-water equations on unstructured mesh consisting of quadrilateral elements. In this paper the algorithm of the finite-volume method is discussed in detail and particular attention is paid to accurately representing the complex irregular computational domain. The lower Yellow River reach from Huayuankou to Jiahetan is a typical meandering river. The generation of the computational mesh, which is used to simulate the flood, is affected by the distribution of water works in the river channel. The spatial information about the two Yellow River levee, the protecting dykes, and those roads that are obviously higher than the ground, need to be used to generate the computational mesh. As a result these dykes and roads locate the element interfaces of the computational mesh. In the model the finite-volume method is used to solve the shallow-wave equations, and the Osher scheme of the empirical function is used to calculate the flux through the interface between the neighbouring elements. The finite-volume method has the advantage of using computational domain with complex geometry, and the Osher scheme is a method based on characteristic theory and is a monotone upwind numerical scheme with high resolution. The flood event with peak discharge of $15300 \mathrm{~m}^{3} / \mathrm{s}$, occurring in the period from 30 July to 10 August 1982, is simulated. The estimated result indicates that the simulation method is good for routing the flood in a region with complex geometry. Copyright (c) 2002 John Wiley \& Sons, Ltd.
\end{abstract}

KEY WORDS two-dimensional flood simulation; shallow-water equation; Yellow River; irregular and unstructured mesh; finite-volume method; Osher scheme

\section{INTRODUCTION}

Different two-dimensional flood simulation methods, such as the finite-difference method, the finite-element method and the finite-volume method, have different advantages and limitations. Because of the rectangular grid, the classic finite-difference method (FDM) requires a rather fine mesh size in order to give a satisfactory representation for complicated and curved boundary lines, and a rather small time-step, constrained by the wellknown Courant condition (Abbott, 1979; Chaudhry, 1979). All these will lead to a high computational effort. Although the finite-element method (FEM) does not always require a regular mesh, it leads to dense matrices and therefore involves lengthy matrix inversion procedures that cause difficulties in recursive calculations of unsteady flow problems (Bauer and Schmidt, 1983). The finite-volume method (FVM), which has the merits of both FDM and FEM, was apparently introduced first in the field of numerical fluid dynamics, independently by McDonald (1971) and MacCormack and Paullay (1972), for the solution of two-dimensional, time-dependent Euler equations (Hirsch, 1988).

The governing shallow water-wave equations can be solved by the numerical methods that are well established in aerodynamics owing to the similarity between the shallow-water equations and the compressible Euler equations. There are several approaches to handle the velocity-pressure coupling in simulating

* Correspondence to: Hongtao Wan, LREIS, Institute of Geography Science and Resource, CAS, Building 917, Datun Road, Anwai, Beijing 100101, People's Republic of China. E-mail: wanht@ Ireis.ac.cn 
compressive fluid flow, such as the SIMPLE algorithm (Patankar, 1980). The extension of this technique to non-orthogonal grid cases results in a complex pressure correction equation (Peric, 1990). For calculating the fluxes through the element interface, research has focused on developing and solving the flow equations with high-resolution schemes, which include the total variation diminishing (TVD) schemes of Harten (1983), the flux-corrected transport (FCT) methods of Boris and Book (1973), Book et al. (1975), Zalesak (1979) and Scott $e$ t al. (1999) the switches and cell-averaged piecewise fits utilized in the piecewise parabolic method of Collela and Woodward(1984), and the essentially non-oscillatory (ENO) schemes of Harten and Osher (1987), Osher and Solomone (1982) and Shu and Osher (1988). Applications also show that the scheme of Roe (1981) meets the requirement of high accuracy and computational efficiency (Ye and McCorquodale, 1996).

Following such progress in aerospace Computational Fluid Dynamics (CFD), these methods have been gradually introduced into hydraulics. As these schemes require the estimation of the numerical fluxes at element interfaces, the finite-volume method seems to be particularly and naturally appropriate for their implementation. Unlike the equation transformation method in which errors may be inevitably introduced by the approximation of the Jacobian matrix at each time-step, the finite-volume approach is more accurate and efficient. Alcrudo and Garcia-Navarro (1993) reported a Godunov-type upwind method implemented in finitevolume form using an irregular grid for hydraulic flows. Zhao et al. (1994) and Cheng et al. (1995) presented a two-dimensional unsteady-flow model that is based on the finite-volume method with a combination of unstructured triangular and quadrilateral grids in a river-basin system, and their results were encouraging.

In areas of complex landform, such as the floodplain and complex river channels, there exist many habitats, small dykes, roads and ditches, that constrict greatly the area influenced by moderate and small flood events. When the peak discharge is large enough to break the river levee, the ditch dyke and road located in the region would restrict the movement of the floodwater because the spatial distribution of these elements has a great effect on the flood process. Because the consideration of the spatial distribution of these structures in mesh generation would provide great difficulty in the computational domain discretization and flood simulation effort, these structures were usually ignored in the flood simulation, based on the assumption that the computational domain is simple and smooth. If the spatial distribution of such structures is not considered in the mesh generation, the estimated flood process would be greatly different to the observed one. An irregular and unstructured quadrilateral has the advantage of representing the computational domain with complex geometry and background. The information about banks, dykes and roads can be used to generate this type of computational mesh. Consequently the dykes and roads are located in the element interface of the computational mesh.

This paper presents a two-dimensional flood simulation model, which is written in Visual C++ language and adopts the FVM with the Osher scheme for solving a Riemann problem and an empirical equation of a weir or other hydraulic structure for calculating the numerical fluxes through the element interfaces where the flood control structures are located. In this two-dimensional model the two-dimensional flow process is described by the depth-averaged, shallow water-flow equations, and the computational domain is represented by an irregular and unstructured grid. We used the flood model to simulate and analyse the extreme flood event that occurred in August 1982, through which we obtain more detailed information about the hydrological and hydraulic characteristics in the lower Yellow River. The information about water stage can be used to guide the withdrawal of the victims and evaluate the disaster.

\section{GOVERNING EQUATIONS}

The conservation form of the two-dimensional shallow-water equations is given by the mass equation

$$
\frac{\partial h}{\partial t}+\frac{\partial(h u)}{\partial x}+\frac{\partial(h v)}{\partial y}=0
$$


and momentum equations

$$
\begin{gathered}
\frac{\partial(h u)}{\partial t}+\frac{\partial(h u u)}{\partial x}+\frac{\partial(h u v)}{\partial y}+\frac{\partial\left(g h^{2} / 2\right)}{\partial x}=g h\left(S_{0 x}+S_{\mathrm{fx}}\right) \\
\frac{\partial(h v)}{\partial t}+\frac{\partial(h u v)}{\partial x}+\frac{\partial(h v v)}{\partial y}+\frac{\partial\left(g h^{2} / 2\right)}{\partial y}=g h\left(S_{0 y}+S_{\mathrm{f} y}\right)
\end{gathered}
$$

where $h$ is water depth, $u$ and $v$ are depth-averaged velocity components in $x$ and $y$ directions, respectively, $g$ is gravitational acceleration, $S_{0 x}$ and $S_{\mathrm{f} x}$ are bed slope and friction slope in the $x$ direction, and $S_{0 y}$ and $S_{\mathrm{f} y}$ are bed slope and friction slope in the $y$ direction.

The mass and momentum equations can be written in vector form

$$
\frac{\partial q}{\partial t}+\frac{\partial f(q)}{\partial x}+\frac{\partial g(q)}{\partial y}=\boldsymbol{b}(\boldsymbol{q})
$$

where

$$
\boldsymbol{q}=\left[\begin{array}{c}
h \\
h u \\
h v
\end{array}\right] ; \quad \boldsymbol{f}(\boldsymbol{q})=\left[\begin{array}{c}
h u \\
h u^{2}+g h^{2} / 2 \\
h u v
\end{array}\right] ; \quad \boldsymbol{g}(\boldsymbol{q})=\left[\begin{array}{c}
h v \\
h u v \\
h v^{2}+g h^{2} / 2
\end{array}\right] ; \quad \boldsymbol{b}(\boldsymbol{q})=\left[\begin{array}{c}
0 \\
g h\left(S_{0 x}-S_{f x}\right) \\
g h\left(S_{0 y}-S_{f y}\right)
\end{array}\right]
$$

are conserved physical vectors and flux vectors in the $x$ and $y$ directions.

\section{Basic equation of the finite-volume method (FVM)}

Upon integrating Equation (4) over an arbitrary element $\Omega$, the basic equation of FVM can be derived by using the divergence theorem

$$
\iint_{\Omega} q_{t} d \omega=-\int_{\partial \Omega} F(\boldsymbol{q}) \times \boldsymbol{n} d L+\iint_{\Omega} b(\boldsymbol{q}) d \omega
$$

where $\boldsymbol{n}$ is a unit outward vector normal to the boundary, and $d \omega$ and $d L$ are area and arc elements, respectively. The integrand $F(\boldsymbol{q}) \times n$ is normal flux vector in which $F(\boldsymbol{q})=[\boldsymbol{f}(\boldsymbol{q}), \boldsymbol{g}(\boldsymbol{q})]^{T}$. The resolution of the flux vector in the normal direction allows treatment of the two-dimensional problem as a local one-dimensional problem.

The vector quantity $\boldsymbol{q}$ is assumed uniform in any element. Thus, discretizing Equation (6), the basic equation of FVM is

$$
A \frac{\mathrm{d} q}{\mathrm{~d} t}=-\sum_{j=1}^{m} F_{n}^{j}(\boldsymbol{q}) L^{j}+A \boldsymbol{b}(\boldsymbol{q})
$$

where $A$ is area of an element, $m$ is the total node number of an element, $j$ is the index of the element side, $L^{j}$ is the length of the side, and $\boldsymbol{b}(\boldsymbol{q})$ is the source (or sink) term of Equation (4). Each side of the element, $F_{n}^{j}(\boldsymbol{q})$, which is denoted hereafter by $F_{n}(\boldsymbol{q})$, is defined as (Spekreijse 1988)

$$
F_{n}(\boldsymbol{q})=\cos \Phi \boldsymbol{f}(\boldsymbol{q})+\sin \Phi \boldsymbol{g}(\boldsymbol{q})
$$

where $\Phi$ is the angle between the normal direction of vector $\boldsymbol{n}$ and the $x$ axis (measured counterclockwise from the $x$ axis). For simplifying the calculation process, Spekreijse (1988) introduced the transformation matrices $\boldsymbol{T}(\Phi)$, which represent the rotation of coordinate axes

$$
\mathbf{T}(\Phi)=\left[\begin{array}{ccc}
1 & 0 & 0 \\
0 & \cos \Phi & \sin \Phi \\
0 & -\sin \Phi & \cos \Phi
\end{array}\right]
$$


It is obvious that $\mathbf{T}(\Phi)$ is the $\mathbf{H}$ matrix and meets the relationship $\mathbf{T}(\Phi)^{-1}=\mathbf{T}(\Phi)^{T}$, then

$$
\begin{aligned}
\boldsymbol{T} \Phi F_{n}(\boldsymbol{q}) & =\left[\begin{array}{ccc}
1 & 0 & 0 \\
0 & \cos \Phi & \sin \Phi \\
0 & -\sin \Phi & \cos \Phi
\end{array}\right] \times\left[\begin{array}{c}
h(u \cos \Phi+v \sin \Phi) \\
h(u \cos \Phi+v \sin \Phi)^{2}+g h^{2} / 2 \\
h(u \cos \Phi+v \sin \Phi)(-u \sin \Phi+v \cos \Phi)
\end{array}\right] \\
& =\left[\begin{array}{c}
h(u \cos \Phi+v \sin \Phi) \\
h(u \cos \Phi+v \sin \Phi)^{2}+g h^{2} / 2 \\
h(u \cos \Phi+v \sin \Phi) \times(-u \sin \Phi+v \cos \Phi)
\end{array}\right] \\
& =\boldsymbol{f}[\mathbf{T}(\Phi) \boldsymbol{q}]=\boldsymbol{f}(\overline{\boldsymbol{q}})
\end{aligned}
$$

where $\overline{\boldsymbol{q}}=\mathbf{T}(\Phi) \boldsymbol{q}$, and through this transform the conservational vector $\mathbf{q}$ can be calculated by

$$
F_{n}(\boldsymbol{q})=\mathbf{T}(\Phi)^{-1} \boldsymbol{f}(\overline{\boldsymbol{q}})
$$

Based on the Equation (11), Equation (7) can be rewritten as

$$
A \frac{\mathrm{d} q}{\mathrm{~d} t}=-\sum_{j=1}^{m} \mathbf{T}(\Phi)^{-1} \boldsymbol{f}(\overline{\boldsymbol{q}}) L^{j}+A \boldsymbol{b}(\boldsymbol{q})
$$

Note that the quantity $\bar{q}$ is derived from $\mathbf{q}$, with velocity components in the normal and tangential directions. The quantity $\mathbf{q}$ or $\overline{\boldsymbol{q}}$ can have different values in each element in the computational domain and thus the value of $\mathbf{q}$ is discontinuous across the interface between elements. Because of this discontinuity, the estimation of the transformed flux $\mathbf{f}(\overline{\boldsymbol{q}})$ in Equation (12) can be simplified into a problem of solving a local one-dimensional Riemann problem in the normal direction of the element interface.

\section{ESTIMATION OF THE NORMAL-FLUX-RIEMANN PROBLEM}

The governing equation of the one-dimensional normal-flux-Riemann problem is

$$
\overline{\boldsymbol{q}}_{t}+[\mathbf{f}(\overline{\boldsymbol{q}})]_{\bar{x}}=0
$$

in which the origin of the $\bar{x}$ axis is located in the mid-point of the element interface and the mass and momentum flux flow along the normal direction. Because there are three equations in Equation (13), it is different from the true one-dimensional Riemann problem. When $t$ is 0 we should solve the one-dimensional Riemann problem to obtain the result of $\overline{\boldsymbol{q}}_{L}$ and $\overline{\boldsymbol{q}}_{R}$, when $\overline{\boldsymbol{q}}_{L}$ is $\overline{\boldsymbol{q}}_{L}$ if $\bar{x}$ is less than 0 and $\overline{\boldsymbol{q}}_{L}$ is $\overline{\boldsymbol{q}}_{R}$ if $\bar{x}$ is more than 0. Solving this Riemann problem we can obtain the $F_{L R}\left(\overline{\boldsymbol{q}}_{L}, \overline{\boldsymbol{q}}_{R}\right)$, which is the normal mass and momentum flux, in the $\bar{x}-\bar{y}$ coordinate system. Through solving Equation (10) we obtain the normal flux $\mathbf{f}_{\mathrm{n}}(\mathbf{q})$ in the $x-y$ coordinate system.

In our application the Osher scheme was applied to solve the Riemann problem in order to obtain the normal flux $F_{\mathrm{LR}}$. For simplification we omit the sign '-' in Equation 13 in the following discussion. Firstly we can describe the characteristic of Equation (13), where the Jacobian matrix A of Equation (13) is

$$
\mathbf{A}=\frac{\mathrm{d} f}{\mathrm{~d} q}=\left[\begin{array}{ccc}
0 & 1 & 0 \\
c^{2}-u^{2} & 2 u & 0 \\
-u v & v & u
\end{array}\right]
$$

in which $c$ is the square root of $g \times h$. The solution of the characteristic equation, $|\mathbf{A}-\lambda I|=0$, and the eigenvector equation, $A \times r_{k}=\lambda_{k} \times r_{k}$, are

$$
\begin{aligned}
\lambda_{1}=u-c & \lambda_{2}=u \quad \lambda_{3}=u+c \\
r_{1} & =(1, u-c, v)^{T} \quad r_{2}=(0,0,1)^{T} \quad r_{3}=(1, u+c, v)^{T}
\end{aligned}
$$


where $\lambda_{1}, \lambda_{2}, \lambda_{3}$ are the eigenvalues of the characteristic equation and $r_{1}, r_{2}, r_{3}$ are the eigenvectors of the eigenvector equations. Based on the eigenfunction $\mathrm{d} x / \mathrm{d} t=\lambda_{\mathrm{k}}$ with eigenvectors $r_{\mathrm{k}}$ and eigenvalues $\lambda_{\mathrm{k}}$, the Riemann invariant $J_{k}(\boldsymbol{q})$ can be defined as $\nabla_{q} J_{k} \times r_{k}=0$. By solving this equation the components of the Riemann invariant are obtained

$$
\begin{array}{lll}
\lambda_{1}: & J_{1}^{(1)}=u+2 c=\mathrm{constant}, & J_{1}^{(2)}=v=\mathrm{constant} \\
\lambda_{2}: & J_{2}^{(1)}=u=\mathrm{constant}, & J_{2}^{(2)}=h=\mathrm{constant} \\
\lambda_{3}: & J_{3}^{(1)}=u-2 c=\mathrm{constant}, & J_{3}^{(2)}=v=\mathrm{constant}
\end{array}
$$

These Riemann invariants remain constant along their corresponding characteristic curve and the superscripts 1 and 2 indicate the two Riemann invariants for each eigenvector.

Deriving from the character of the Riemann invariant we obtain the following equations

$$
\begin{array}{ll}
u_{L}+2 c_{L}=u_{A}+2 c_{A}, & v_{L}=v_{A} \\
u_{A}=u_{B}, & h_{A}=h_{B} \\
u_{R}+2 c_{R}=u_{B}+2 c_{B}, & v_{R}=v_{B}
\end{array}
$$

solutions of the equations are

$$
\begin{aligned}
& u_{A}=u_{B}=\left(J_{L}+J_{R}\right) / 2 \\
& h_{A}=h_{B}=\left(J_{L}-J_{R}\right)^{2} /(16 g)
\end{aligned}
$$

where

$$
J_{L}=u_{L}+2 c_{L}, \quad J_{R}=u_{R}-2 c_{R}
$$

According to the sign of the eigenvalues, the flux $f(q)$ can be divided into forward and backward flux components (Spekreijse, 1988)

$$
\boldsymbol{f}(\boldsymbol{q})=\boldsymbol{f}^{+}(\boldsymbol{q})+f^{-}(\boldsymbol{q})
$$

The approximate solution of the Riemann problem is

$$
\begin{aligned}
F_{L R}\left(\boldsymbol{q}_{L}, \boldsymbol{q}_{R}\right) & =\boldsymbol{f}^{+}\left(\boldsymbol{q}_{L}\right)+\boldsymbol{f}^{-}\left(\boldsymbol{q}_{R}\right) \\
& =\boldsymbol{f}\left(\boldsymbol{q}_{L}\right)+\int_{q_{L}}^{q_{R}} \mathbf{A}^{-}(q) \mathrm{d} q \\
& =\boldsymbol{f}\left(\boldsymbol{q}_{R}\right)-\int_{q_{L}}^{q_{R}} \mathbf{A}^{+}(q) \mathrm{d} q
\end{aligned}
$$

where $\mathbf{A}^{ \pm}(q)$ are Jacobian matrices of the positive and negative eigenvalues and $\boldsymbol{f}^{ \pm}(\boldsymbol{q})$ are the component fluxes propagating in the positive and negative characteristic directions.

According to the sign of $\lambda$, yielding

$$
F_{L R}\left(\boldsymbol{q}_{L}, \boldsymbol{q}_{R}\right)=\left\{\begin{array}{cc}
\boldsymbol{f}\left(\boldsymbol{q}_{1}\right) & (\lambda \geq 0) \\
\boldsymbol{f}\left(\boldsymbol{q}_{2}\right) & (\lambda \leq 0) \\
\boldsymbol{f}\left(\boldsymbol{q}_{2}\right)-\boldsymbol{f}\left(\boldsymbol{q}_{3}\right)+\boldsymbol{f}\left(\boldsymbol{q}_{1}\right) & \left(\lambda\left(q_{1}\right)>0, \lambda\left(q_{2}\right)<0\right) \\
\boldsymbol{f}\left(\boldsymbol{q}_{3}\right) & \left(\lambda\left(q_{1}\right)<0, \lambda\left(q_{2}\right)>0\right)
\end{array}\right.
$$

\section{APPLICATION AND RESULTS}

Study area

The Yellow River basin is greatly influenced by its typical seasonal climate of obvious wet season and dry seasons sixty to eighty per cent of annual rainfall amounts, which usually is in the form of heavy rainstorms, occur from July to October. Because the channel flow of the Yellow River is generated mainly 
from precipitation the uneven yearly distribution of rainfall determined that the flow was irregularly distributed in a year. The flow recorded at river gauging stations in the wet season is $60 \%$ of the annual flow. The flow amounts from March to June, which is dry season, represents only $10-20 \%$ of the annual flow. As an example, at Huayuankou gauging station the flow amounts from July to October are 57.8\% of total annual flow, but only $17.4 \%$ is recorded from April to June, when it is dry season and water demand is very high.

Floods in the lower Yellow River are generated mostly from heavy rainstorm of short duration and high intensity. One flood water source region is located in the subcatchment between Hekouzhen township and Sanmenxia Dam and the other is between Sanmenxia Dam and Huayuankou gauging station, of which the total area influenced by rainstorm is usually $20000-30000 \mathrm{~km}^{2}$. Heavy rainstorm events in the two subcatchments have never occurred at the same time. So extreme floods have the characteristics of a high water stage, low runoff amounts and short duration, which usually is less than 12 days and is even less than 5 days sometimes (Wu et al., 1987).

The lower Yellow River reach from Huayuankou to Jiahetan is a typical meandering river and has the reputation of 'the river over the ground' in the world. Its length is $83.1 \mathrm{~km}$, the distance between the two Yellow River levee varies from 5 to $20 \mathrm{~km}$, the width of the main river channel varies from 3.2 to $4.5 \mathrm{~km}$. The gradient along the main river channel centre line varies from 0.00017 to 0.00027 and its average value is about $0 \cdot 0002$. The floodplain is usually $2 \mathrm{~m}$ higher than the main river channel bed, and lower than $1 \mathrm{~m}$ in some place Figure 1. The riverbed is 3-5 m higher than the ground beyond the two Yellow River levee and is more than $10 \mathrm{~m}$ in some river reaches. The average sinuosity of this river reach is about $1 \cdot 15$. Because the riverbed is mainly made of sand and silt the river channel is wide and shallow with many rapids and shoals and always changes its main course. By ground elevation the floodplain can be divided into three types: high, middle and low (Bing, 1987).

The flood events occurring in the Yellow River usually breach the riverbed and even make the river change its main channel. There have been about 1500 cases of dyke-break during the 2000 years before 1949 (Xu, 1989). Owing to good management in the several decades after 1949 there have been few dyke-break events since. However, the river reach from Huayuankou to Jiahetan has a high probability of dyke-break. So the two-dimensional flood model is necessary to develop in order to explore the hydrological characteristics of the river flow, understand the flood process and obtain information about water stage, which can be used to guide the withdrawal of flood victims and evaluate the disaster.

In the early August 1982 an extreme flood event occurred in the lower Yellow River, the second largest extreme flood event in the 40 years since 1958, and is the most extreme flood event with the highest peak flow since the Sanmenxia Dam was built. The peak flow discharge in Huayuankou observation station is $15300 \mathrm{~m}^{3} / \mathrm{s}$, and has the characteristics of large flow discharge and relatively low sediment concentration.

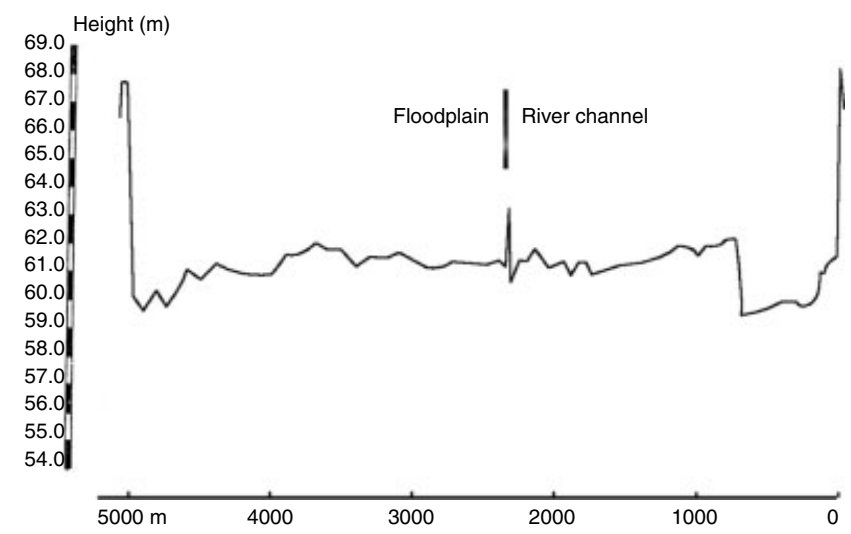

Figure 1. The Yellow River cross-section at Huayuankou gauging station 


\section{Computational mesh}

In the finite-volume algorithm the conservative forms of the two-dimensional shallow water-wave equations were discretized upon the computational domain, which can be represented by various irregular unstructured computational meshes, with square or arbitrary geometry. The structure of the model changed little with different types of mesh. In most cases these mesh are geometrically fairly simple. The three node triangle and the four node quadrilateral, especially the trapezium, are commonly used to generate the irregular unstructured computational mesh. The advantage of an unstructured irregular computational mesh is that it can fit the boundary of a computational domain very well. The boundary conditions are easy to be dealt with because the computational mesh generated has taken account of the location and distribution of the water control projects, irrigation works and roads. The computational mesh is easily modified and adjusted to change its form and density and more easily generated than other meshes. For example, the triangle irregular network can be generated automatically. In our application we used a trapezium, which is complemented by some three- and five-node polygons (Figure 2).

\section{The topography and land use in the Yellow River channel}

For constructing the flood simulation model we need information on topography, land use, hydrology, meteorology and climate, water conservancy projects and facilities, and flood management facilities. To generate the model for simulating the flood in the river reach from Huayuankou to Jiahetan the following information was used: (i) map of water works and other facilities influencing the floodplain (Figure 3); (ii) topography map; (iii) land-use map (Figure 4); and (iv) observed hydrological data at the Huayuankou and Jiahetan hydrological gauging stations.

All the maps are of the lower Yellow River and its floodplain and most are at a scale of 1:50000, and were provided by the Yellow River Conservancy Commission.

The distance between the two Yellow River levee is very wide. In the dry season, runoff is low and so are the peaks of most flood events. As a result, many types of habitat are distributed in the floodplain. To protect these habitats many little dykes, called protecting dykes, with irregular orientation, have been constructed. In addition, there are many roads and ditches that are 1-2 $\mathrm{m} \mathrm{high/deep,} \mathrm{which} \mathrm{constrict} \mathrm{greatly}$

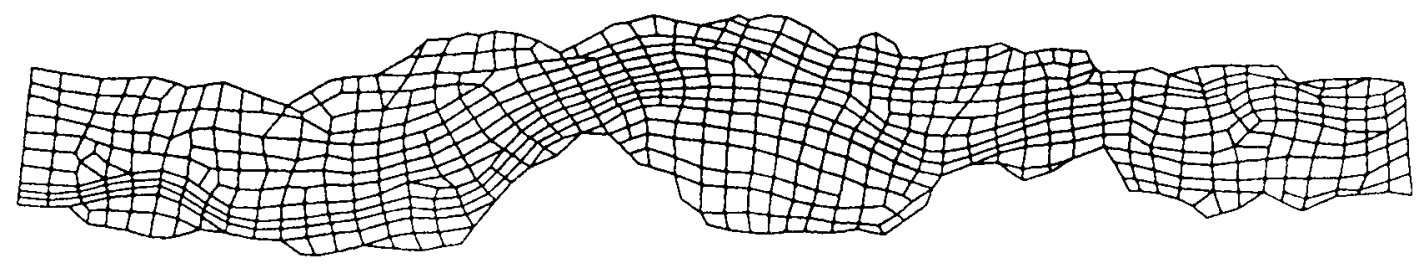

Figure 2. The computational mesh

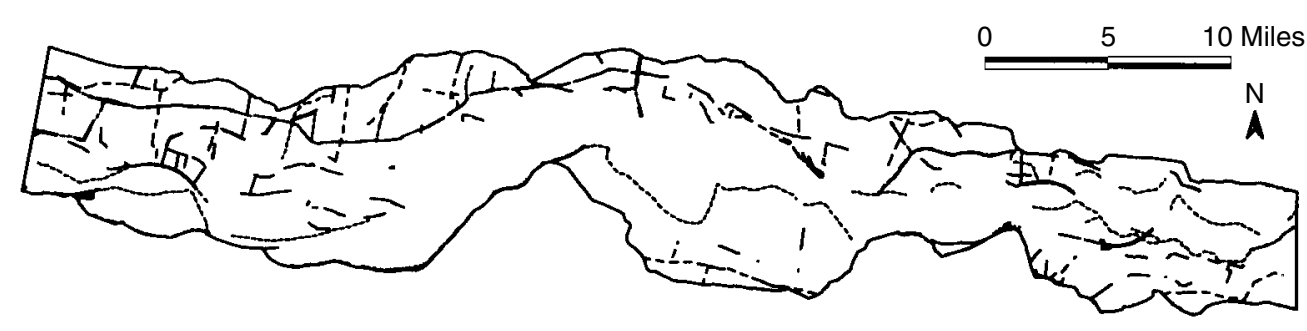

$\wedge$ Irrigation works $\square$ Boundary $/$ Dyke $\therefore$ Ditch $\cong$ Road

Figure 3. Map of flood control structures 


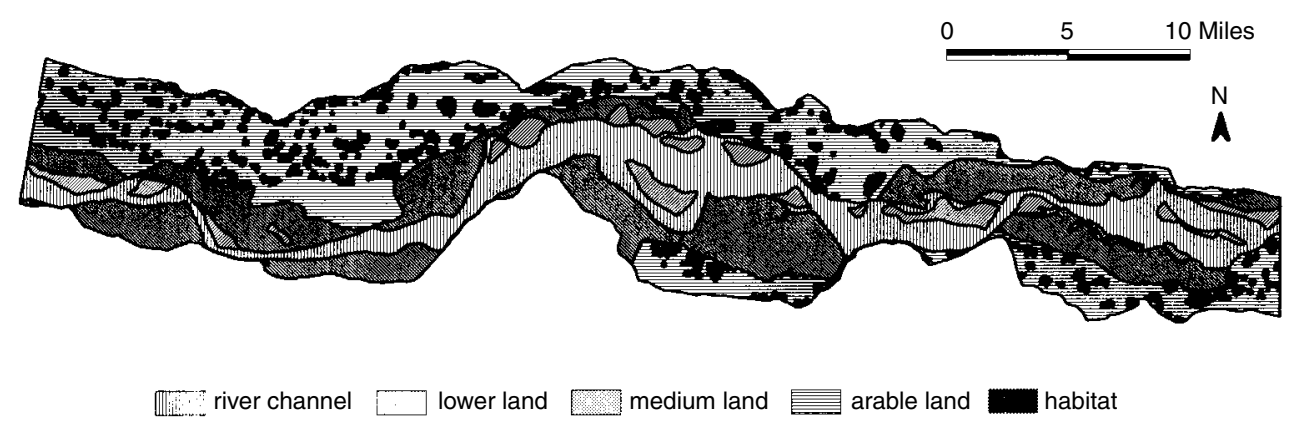

Figure 4. The land-use map of lower Yellow River

the area influenced by medium and small flood events. If the peak discharge is large enough the floodwater would break these protecting dykes, inundate those habitats located between the two Yellow River levee, and cause great loss to people living there. The generation of the computational mesh to be used to simulate the flood in the river reach from Huayuankou to Jiahetan, is affected by the distribution of water works in the floodplain. Information about the two Yellow River levee, the protecting dykes, and those roads that are obviously higher than the ground, is needed in order to generate the computational mesh for simulating flood events. Accordingly these dykes and roads are located in the element interface of the computational mesh.

The structure of the computational mesh allows each element to have a different roughness coefficient, although usually this is not practical owing to the large data requirements. In most applications, the parameters are measured for each soil type or region with different surface conditions and it is assumed that these parameters do not vary within each region or soil type. A map of the regions of different parameters can then be overlaid numerically on the mesh element map of the computational domain. This approach is based on the assumption that the variation in parameter values within a region is less than that between regions and that the within element characteristics are uniform, therefore the soil texture dominates the roughness parameter values.

The land use of the computational domain can be classified simply into five categories, which are habitat, arable land, medium land, lower land and main river channel, respectively. Different land-use types present different resistance to the river flow with low water depth, especially with a water depth of less than $1 \mathrm{~m}$. Based on past simulation application and roughness measure experiment the correlation of land-use type with roughness coefficient was assumed: the roughness parameters (Manning's $n$ values) were estimated from the field as 0.07 for the habitat, 0.06 for the arable land, 0.05 for the medium land, 0.03 for the lower land and 0.01 for the river channels. Based on this assumption the land-use map is converted into the roughness parameter map. The computational mesh is overlaid on the roughness parameter map to obtain the roughness parameters in every computational element by the area-weighted interpolation method. Similarly, the computational mesh is overlaid on the $30 \times 30 \mathrm{~m}$ digital elevation model $(\mathrm{DEM})$, which is generated from the topography map and individual elevation points at the scale of 1:50000, to obtain the elevation value in every computational element by the area-weighted interpolation method. The element interface is assigned the elevation value of the corresponding dyke elevation, however, if there is not a dyke or road the element interface is assigned the higher elevation value of the two neighbouring computational mesh centres.

The riverbed roughness has a inverse relationship with water depth, and most simulation applications indicate that the roughness coefficient should be changed gradually and smoothly in the simulation process. If the water depth is less than $1 \mathrm{~m}$ the roughness is assumed to have a reciprocal correlation with the water depth or the square of water depth. The increasing roughness can prevent the sudden increase of estimated velocity, so it is beneficial for simulation stability. When the water depth is very low, excess roughness, which can produce negative velocity or even make the simulation unstable, should be avoided. In our application we assumed that the roughness has a reciprocal correlation with the square of water depth if the water depth is less than $1 \mathrm{~m}$. 


\section{INITIAL CONDITIONS AND BOUNDARY CONDITIONS}

\section{Initial conditions}

Before simulation the initial value, including initial velocity components in the $x$ and $y$ directions, water stage and water depth, etc., should be assigned to every computational element. In our application of simulating flood events in the Yellow River, velocity components in the $x$ and $y$ directions in every mesh unit are assumed to be 0 and the water depth in every mesh unit is assumed to be $0.5 \mathrm{~m}$, water stage is the sum of water depth and the altitude of the riverbed. Then the convective terms in the governed equations were omitted and the discharge in the inlet is given the same initial value in $24 \mathrm{~h}$. The result of simulating the steady state river flow was assigned as the initial value (velocity components in the $x$ and $y$ directions, water stages and water depth)

\section{Boundary conditions}

In the two-dimensional flood simulation model, there are three types of boundary condition, which are boundary condition of solid walls, transmissive boundary for subcritical flow and interior boundary, all these boundary conditions are assigned at the element interface.

Solid boundary. The solid boundary is also called a closed boundary. If an interface between two elements is a solid boundary there is no flow across that interface, so there is no mass flux. For this type of boundary condition it is critical to handle the momentum loss in the interface between the water and land. In our application we correct static water pressure in the interface between the water and land with the dynamic water pressure and assume that the corrected water pressure is equal to the sum of the static water pressure of the element and the normal velocity:

$$
h^{\prime}=\sqrt{h u|u|+g h^{2} / 2}
$$

where $h$ is the water depth (static water pressure) in the boundary and $u$ is the velocity component of the element centre in the normal direction and $h^{\prime}$ is the corrected static water pressure (water depth).

Open boundary. When an element interface coincides with the boundary of the domain or some physical boundary it is necessary to solve a boundary Riemann problem. In riverbed and floodwater storage regions the inlet and outlet are open boundaries. Generally the observed discharge hydrograph can be assigned to the inlet and the observed stage hydrograph or the stage-discharge curve can be assigned to the outlet of the computational domain. In our application in the lower Yellow River the observed flow discharge hydrograph is assigned to the inlet and the observed stage hydrograph is assigned to the outlet downstream.

Interior boundary. In the case of a hydraulic structure, although the calculation is done under the framework of the finite-volume method, the normal fluxes across the interface are not calculated by the Osher scheme. The procedures for estimating normal fluxes of hydraulic structures are described as follows.

Mass flux. The estimation of the mass flux essentially involves calculation of the unit discharge, denoted by $q_{\mathrm{m}}$, across the element interface that forms the interior hydraulic structure. The discharge formulae available in the model depend on the type of hydraulic structure, such as a well, gate-spillway, levee, bridge, or culvert (Zhao et al., 1994). For flow through a weir, a weir formula is used. For the gate-spillway structure, three kinds of flow condition are possible. Namely, free uncontrolled flow (when gates are fully open and the upper pool is controlled by the submergence effect on the tail-water), free controlled flow (when gates are partially open and the pool is unaffected by the tail-water), and submerged controlled flow (when gates are partially open and the upper pool is controlled by the submergence effect of tail-water). Flow through a culvert or a bridge is calculated by empirical equations developed for flow through a culvert. As for the levee, when the 
water level is lower than the top elevation of the levee, the levee is treated as a land boundary, otherwise the formula derived from the weir equation is used.

Momentum fluxes. For the interior boundary, the two components of the momentum flux (the second and third term of the vector $\boldsymbol{f}(\boldsymbol{q})$ in Equation (22) can be calculated simply as follows

$$
\boldsymbol{f}(\overline{\boldsymbol{q}})=\left[\begin{array}{c}
q_{\mathrm{m}} \\
q_{\mathrm{m}} u_{\mathrm{n}}+\frac{g h^{2}}{2} \\
q_{\mathrm{m}} v_{\mathrm{t}}
\end{array}\right]
$$

where $h$ is water depth, $u_{\mathrm{n}}$ and $v_{\mathrm{t}}$ are velocity components in the normal and tangential directions, respectively. These velocity components are defined as $u_{\mathrm{n}}=u \times \cos \Phi+v \times \sin \Phi$ and $v_{\mathrm{t}}=v \times \cos \Phi-u \times \sin \Phi$, in which $u$ and $v$ are the flow velocity component in the $x$ and $y$ directions, respectively.

\section{Simulation results and discussion}

In our application the selected computational domain is the Huanghe River reach from Huayuankou gauging station to Jiahetan gauging station, which are $83.1 \mathrm{~km}$ apart. The computational element elevation is derived from the 1:50000 relief map. The location and area of villages the location and elevation of roads and ditch dykes are derived from the water engineering map provided by the Yellow River Conservancy Commission. Because of its low resistance to the water flow, roads and ditch dykes lower than $0.5 \mathrm{~m}$ are ignored in constructing the computational mesh; however, roads and ditch dykes higher than $0.5 \mathrm{~m}$ are assigned as element interfaces because of their strong resistance to water flow. In the floodplain the dykes, which are composed of the loose earth, are breached by gaps of different width, through which the water can flow. The higher level roads, which are composed of good construction material, cannot be breached so the floodwater flows over the road if the floodwater level is higher than the road surface. As village areas are assumed to be enclosed by cofferdams higher than the floodwater, their area must be removed from the mesh unit.

The simulated flood event occurred in the period from 30 July to 10 August 1982 . The computational timestep is $30 \mathrm{~s}$. Figure 5 shows the estimated stage hydrograph of mesh 90 in the computational domain and the observed stage hydrograph for the inlet and outlet. The result indicates that the estimated stage hydrograph has the same increasing and decreasing trend as the observed value at the inlet and outlet. The occurrence sequence of the flood peak, of which the flood peak in mesh 90 occurred earlier than the outlet mesh and later than the inlet mesh, is reasonable. Figure 6 is the estimated velocity field of the computational domain in the Yellow River reach from Huayuankou to Jiahetan hydrological gauging station. That the velocity direction of the flow in the channel is along the main channel indicates that the estimated velocity field is reasonable.

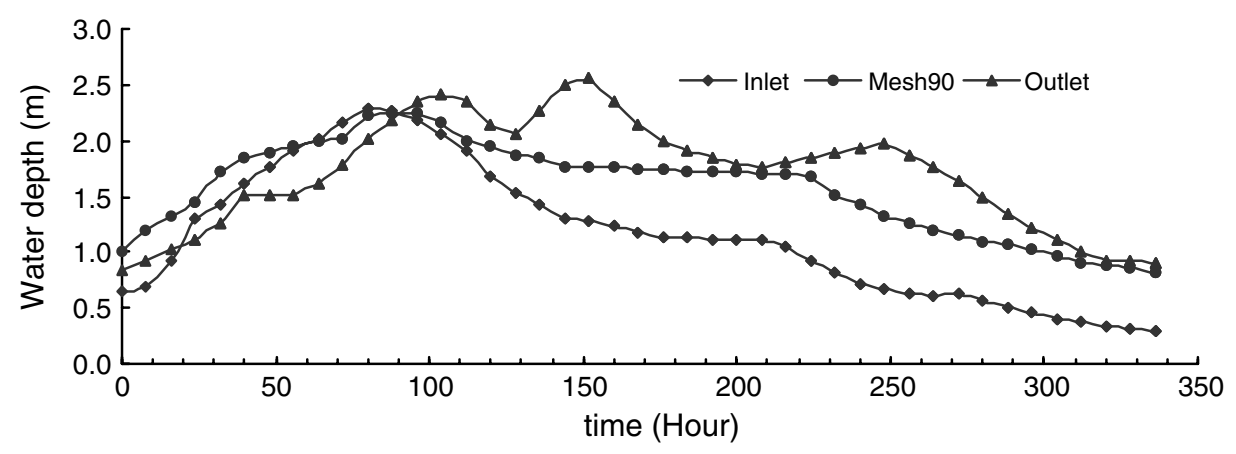

Figure 5. The estimated stage hydrograph compared with observed stage hydrographs at the inlet and outlet 


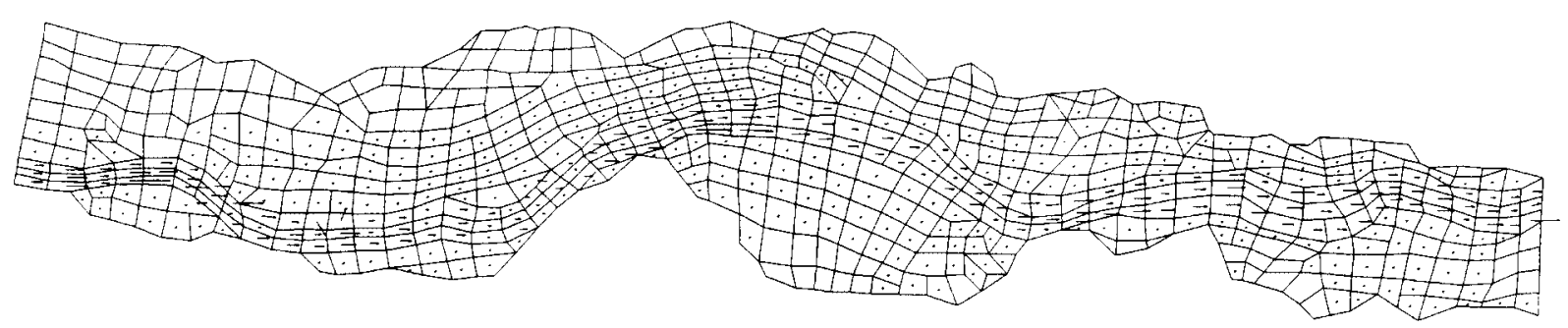

Figure 6. The estimated velocity field

From the foregoing discussion, the model appears to have the capability to simulate the flood process in a complex floodplain region. The advantages and disadvantages of our algorithm are as follows:

1. The computational mesh is a two-dimensional irregular and unstructured mesh, which can allow for the location and distribution of the water works and roads. The irregular and unstructured mesh can represent the real computational domain, in which the roads and dykes that can stop and resist the floodwater flow can be assigned as the mesh interface. As a result the simulated flow field in the floodplain is more practical. In the region with greater relief change the finer mesh can represent the real topography.

2. In comparison with the one-dimensional flood model, the two-dimensional flood model with irregular and unstructured mesh can simulate the flood process in more detail and the result can provide more information, such as the water depth, water stage and velocity at every point, which the one-dimensional model cannot obtain.

3. The two-dimensional model with irregular and unstructured mesh can represent the topography more truly. A finer mesh is used in regions with higher and more complex relief change and coarser mesh is used to represent regions with lower and more simple relief change, such that a better result of flood simulation can be obtained at less computational cost.

4. In the complex Huanghe River reach, the one-dimensional model or two-dimensional model with regular mesh cannot obtain a suitable or reasonable result, so the simulation application opportunities are few. The reasonable result of our flood simulation application provides some achievement in some aspects.

5. In finite-volume method, the basic principle of the algorithm is that the mass is in balance in every mesh unit, so the mass is balance in the whole computational domain.

6. Because the observational information is incomplete and was obtained at different times, validation of the simulation result by comparison with the observed data is difficult, particularly as simulation results are not available for every point in the landscape. In future flood events we will pay more attention to collecting data in order to validate the simulation result and revise the algorithm. In addition, those model parameters confirmed by one or two flood events have some limited use to predict behaviour in future flood events.

\section{CONCLUSIONS}

The reasonable results of our research indicate that the depth-averaged shallow water-wave equations can simulate a flood that occurred in the Yellow River very well. In the flood simulation model the finite-volume technique is used to solve the two-dimensional shallow-water equation on unstructured and irregular mesh consisting of quadrilateral elements, which is complemented by some three- and five-node polygons. The estimated result with good agreement with the observed data indicates that this simulation method can be applied to the lower Yellow River and its floodplain with complex and arbitrary geometry. In the model the finite-volume method is used to solve the shallow-wave equations and the Osher scheme or an empirical 
equation of hydraulic structures are used to calculate the flux through the interface between neighbouring elements, which is a Riemann problem. The finite-volume method had the advantage of being applied in complex and arbitrary computational domains and the Osher scheme is a method based on characteristic theory and is a monotone upwind high-resolution numerical scheme. In addition, the Osher scheme is a firstorder shock-capturing method in that the smooth flow region and the neighbourhood of discontinuities are handled by the same numerical scheme.

The two-dimensional flood model for simulating a flood event that occurred in the lower Yellow River reach from Huayuankou to Jiahetan was constructed on the basis of fundamental information such as geomorphology, topography maps, waterworks data, hydrological and meteorological data, flood management observations and other relevant data. The flood simulation result shows that the estimated stage hydrograph has the same trend as the observed one at inlet and outlet, with little difference in water depth value. The velocity field of the computational domain is also good. The reasonable result is useful for flood forecasting and planning for post-disaster reaction, because of the information provided about water depth, inundation duration and the velocity field that cannot be achieved by other techniques such as remote sensing.

The sediment concentration of the Yellow River is the highest in the world. In this paper we simulated the hydrological and hydraulic characteristics by simulating a flood event with a large discharge and low sediment concentration. It is a great deficiency to simulate a flood in the Yellow River without the sediment data. In the future we will simulate the flood with low discharge and high sediment concentration using a flood model that takes into account the sediment data as input data or parameters.

\section{ACKNOWLEDGMENTS}

This research was supported by the knowledge innovation project sponsored by the Chinese Academic of Science in P. R. China (CXIOG-D00-05). We express special appreciation to Professor Tan Weiyan and Shi Yong of the Institute of Hydrology in Nanjing, Professor Chen Xiaotao in the Chinese Institute of Water Resources and Hydropower Research for very useful discussions and instructions about implementing the finite-volume method.

\section{REFERENCES}

Abbott MB. 1979. Computational Hydraulics: Elements of the Theory of Free-surface Flows. Pitman: London.

Alcrudo F, Garcia-Navarro P. 1993. A high-resolution Godunov-type scheme in finite volumes for the 2-D shallow water equations. International Journal of Numerical Methods in Fluids 16: 489-505.

Bauer SW, Schmidt KD. 1983. Irregular-grid finite-difference simulation of lake Geneva surge. Journal of Hydraulic Engineering 109(10): $1285-1296$.

Bing Guangmei. 1987. The harness of the wandering lower Yellow River. The People's Yellow River, No. 2. The Yellow River Agency of Shandong Province; 32-36. (In Chinese.)

Book LD, Boris JP, Hain K. 1975. Flux-corrected transport II: generalization of the method. Journal of Computational Physics 18: $248-278$.

Boris JP, Book LD. 1973. Flux-corrected transport I: SHASTA, a fluid transport algorithm that works. Journal of Computational Physics 11: $38-69$

Chaudhry MH. 1979. Applied Hydraulic Transient. Van Nostrand Reinhold: New York.

Cheng X, Yang L, Chen X. 1996. Numerical model of flood propagation in detention basin. Journal of Natural Disasters 5(1): 34-40. (In Chinese.)

Collela P, Woodward PR. 1984. The piecewise parabolic method (PPM) for gas dynamics simulation. Journal of Computational Physics 54: $174-201$.

Harten A. 1983. High-resolution schemes for hyperbolic conservation laws. Journal of Computational Physics 49: $357-393$.

Harten A, Engquist B, Osher S, Chakravarthy SR. 1987. Uniformly high-order-accurate non-oscillatory schemes. Journal of Computational Physics 71(111): 231-303.

Hirsh C. 1998. Numerical Computation of Internal and External Flows, volume 1: Fundamentals of Numerical Discretization. John Wiley \& Sons: New York.

MacCormack RW, Paullay AJ. 1972. Computational Efficiency Achieved by Time Splitting of Finite Difference Operators. American Institute of Aeronautics and Astronautics, Paper 72-154. American Institute of Aeronautics and Astronautics: San Diego, Calif.

McDonald PW. 1971. The Computation of Transonic Flow Through Two-Dimensional Gas Turbine Cascades. American Society of Mechanical Engineers, Paper 71-GT-89. American Society of Mechanical Engineers: New York. 
Osher S, Solomone F. 1982. Upwind difference schemes for hyperbolic systems of conservation laws. Mathematics of Comp. 38: $339-374$. Patankar SV. 1980. Numerical Heat Transfer and Fluid Flow. Hemisphere: Bristol, PA.

Peric M. 1990. Analysis of pressure-velocity coupling on non-orthogonal grids. Numer. Heat Transfer Past B 17(1): 63-82.

Roe PL. 1981. Approximate Riemann solvers, parameter vectors, and difference schemes. Journal of Computational Physics 43(2): $357-372$.

Scott A. Yost, Prasad MSV Rao. 1999. Flux-corrected transport technique for open channel flow. International Journal for Numerical Methods in Engineering 29: 951-973.

Spekreijse SP. 1988. Multigrid Solution of Steady Euler Equations. CWI Tract 46, Center for Mathematics and computer Science, Amsterdam. Shu CW, Osher S. 1988. Efficient implementation of essentially non-oscillatory shock capturing schemes. Journal of Computational Physics 77: 439-471.

The Yellow River Agency of Shandong Province. 1991. The Effect of River Harness on the Eroding and Sediment of the Lower Yellow River Channel. The People's Yellow River, No. 5. 57-60 (In Chinese.)

Zhiyao Wu. 1987. The Flood and its Countermeasures in Lower Yellow River. The People's Yellow River, No. 6. The Yellow River Agency of Shandong Province; 3-7 (In Chinese.)

Fuling Xu. 1989. The evolvement of the lower Yellow River channel. Proceedings of Symposium on Controlling the Flood in the Lower Yellow River. The Chinese Environmental Science Press. (In Chinese.)

Ye J, McCorquodale JA. 1996. Mathematical modelling of mass transport in a curved channel. Proceedings, CSCE Annual Conference, Vol. 1, Canadian Society of Civil Engineering: Edmonton, Alberta; 96-107.

Zalesak ST. 1979. Fully multidimensional flux-corrected transport algorithm for fluids. Journal of Computational Physics 31: 335-362.

Zhao DH, Shen HW, Tabios GQ, Lai JS, Tan WY. 1994. Finite-volume two-dimensional unsteady-flow model for river basins. Journal of Hydraulic Engineering 120(7): 863-883 